\title{
Lilly buys back into the BACE race for Alzheimer's disease
}

Eli Lilly and Company and AstraZeneca have entered into a 50:50 partnership to develop AZD3293, AstraZeneca's $\beta$-secretase (BACE) inhibitor for Alzheimer's disease. Lilly which recently had to drop its own BACE inhibitor LY2886721 owing to liver toxicity - agreed to pay up to US\$500 million and will take the lead on the upcoming Phase II/III trials.

Alzheimer's disease is characterized by the presence of amyloid- $\beta(A \beta)$ plaques in the brain. $A \beta$ is formed from the sequential cleavage of the amyloid precursor protein (APP), first by $\beta$-secretase and then by $\gamma$-secretase. "BACE is probably the best target from a biological perspective because it is the first cleavage of the APP molecule that releases $A \beta$, and $A \beta$ is key to the triggering of Alzheimer's disease." says Eric Karran, Director of

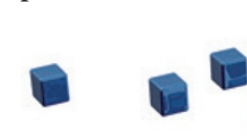
(1)

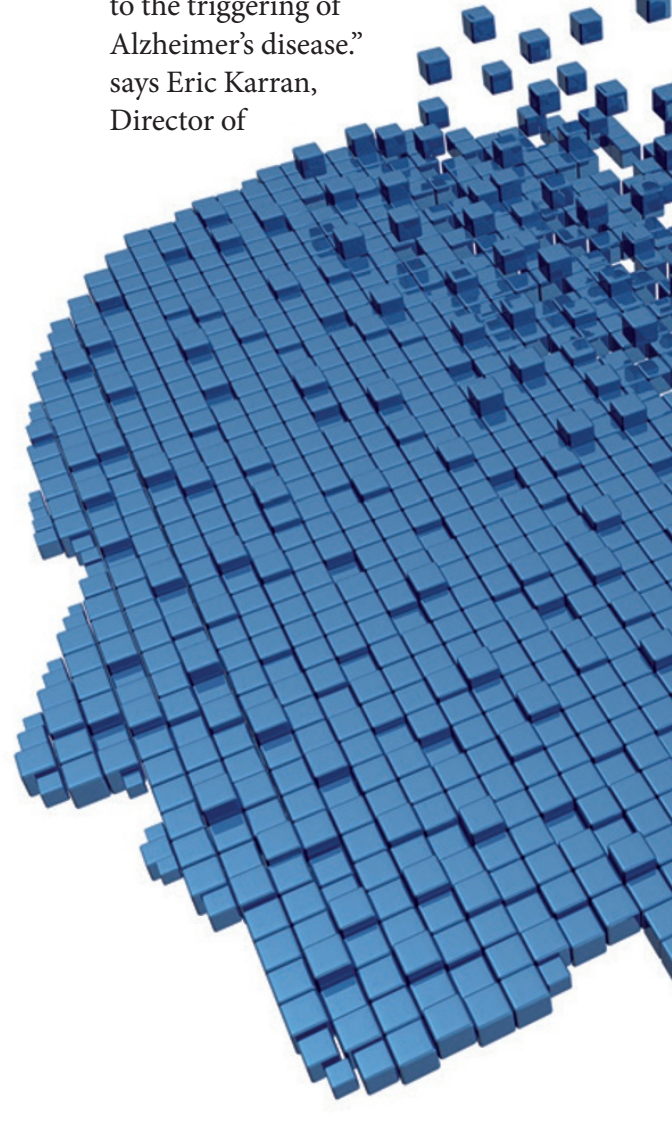

Research at Alzheimer's Research UK, who previously also worked on Lilly's LY2886721.

In the Phase I trial, AZD3293 lowered $\mathrm{A} \beta$ production in humans by up to $75 \%$. "The properties of the molecule, and the amount of $A \beta$ reduction are quite good, and we just couldn't be more pleased to add this compound to our portfolio," says Eric Siemers, Medical Director of Lilly's Alzheimer's disease group.

Treating early in the course of the disease is likely to be key to the success of BACE inhibitors, and more generally of compounds that affect $A \beta$ deposition. Treating patients with moderate or severe forms of Alzheimer's disease with molecules that affect $A \beta$ is

\section{(1)}

"like treating congestive heart failure with a statin," says Steven Paul, CEO of Voyager Therapeutics and the former Executive Vice President for Science and Technology at Lilly. "Amyloid deposition is a very, very early event in the pathogenesis of this disease, and it occurs many years prior to the onset of symptoms," he explains. Paul is also the co-inventor of solanezumab, Lilly's amyloid-specific monoclonal antibody, which is currently in a third Phase III trial in patients with mild Alzheimer's disease and in asymptomatic patients with evidence of $A \beta$ deposition in the hope that it will show effectiveness that it was lacking in two previous Phase III all-comers trials.

"My bet is that these drugs will be more effective when used

$\checkmark$ in combination," says Paul. Karran agrees, highlighting that "combination therapy with two small molecules is actually pretty difficult because you need to make sure that the metabolism, clearance and pharmacokinetics of the drugs are well matched." Combining a small molecule with an antibody would avoid these issues, as antibodies often have half-lives of around 21 days.

Lilly has been heavily involved in Alzheimer's research for many years, and has a second monoclonal antibody for Alzheimer's disease in its pipeline: $\mathrm{mE} 8$, which binds to $A \beta$ plaques. "I think what Lilly's doing is doubling down on Alzheimer's disease," says Paul. "I think it's risky, but the rewards are great."

Both Merck \& Co. and Eisai also have BACE inhibitors in clinical trials; Merck's MK-8931 entered Phase III last November, whereas E2609, developed by Eisai through a partnership with Biogen, is in Phase II. "This is an example where sustained effort — over a decade of work without very much success up until recently - has produced some excellent drugs that we hope will bring relief to an incredibly devastating disease," says Karran.

Megan Cully 